\title{
Docente y aprendiz: enfoques en espacios virtuales
}

\author{
Teacher and Apprentice: Approaches in Virtual Spaces
}

\author{
Natalia Valderrama-Gacitúa ${ }^{1}$
}

\begin{abstract}
RESUMEN
Los espacios virtuales de aprendizaje permiten diversas interacciones entre docente y aprendiz, lo que ha causado debate respecto de la función social y el modelo pedagógico de las instituciones educativas, así como de las competencias que han de tener los/as docentes. La siguiente revisión bibliográfica examina algunos factores claves en la relación docente-aprendiz dentro de espacios virtuales, considerando aspectos afectivos y emocionales, con foco en los efectos de la empatía y su importancia para la "disposición prosocial" del estudiantado, todo lo cual impacta significativamente en las experiencias de aprendizaje.
\end{abstract}

Palabras claves: vínculo docente-aprendiz; espacios virtuales; empatía; tutoría afectiva; conducta prosocial.

\begin{abstract}
Virtual learning spaces allow various interactions between teacher and learner, which has caused debate regarding the social function and pedagogical model of educational institutions, as well as the competences that teachers must have. The following bibliographical review examines some key factors in the teacher-learner relationship within virtual spaces, considering affective and emotional aspects, with a focus on the effects of empathy and its importance for the "prosocial disposition" of students, all of which significantly impacts on learning experiences.
\end{abstract}

Keywords: Teacher-apprentice bond; virtual spaces; empathy; affective tutoring; prosocial behavior.

\footnotetext{
${ }^{1}$ Profesora de Educación Básica, especialidad Matemáticas, Colegio San Esteban Diácono, Santiago, Chile; magíster en Psicología; natalia.gacitua@gmail.com.
} 


\section{El camino de la educación a distancia}

El desarrollo de la educación a distancia en Chile se ha dado en tres etapas. La primera ocurrió a través de la correspondencia, la segunda vino dada por el uso de medios de comunicación, como la televisión y radio, y la tercera se desarrolla con el uso de internet y las tecnologías de la información y la comunicación (TIC). Esta última etapa inició su periodo de expansión a partir del año 2000, cuando los/as docentes generalizaron el uso de las TIC como apoyo de sus clases presenciales y en procesos administrativos En línea con lo anterior, Harmon y Jones (1999) han analizado los diferentes usos educativos que se le daba a la web a finales de los años noventa, organizándolos en niveles jerárquicos (Tabla 1).

\section{Tabla 1}

Niveles de uso educativos de la web

\begin{tabular}{|c|c|}
\hline Niveles & Descripciones \\
\hline Nivel 0 & $\begin{array}{c}\text { No se utiliza en el ámbito educativo o se usa solo para proporcionar información } \\
\text { académica estandarizada. }\end{array}$ \\
\hline Nivel 2 & $\begin{array}{c}\text { Se utiliza de modo complementario al trabajo presencial. La/el profesor/a ofrece } \\
\text { lecturas adicionales o apuntes de la clase. }\end{array}$ \\
\hline Nivel 3 & $\begin{array}{c}\text { El acceso a la web es esencial en el desarrollo del curso. El/la estudiante obtiene de } \\
\text { ahí los principales recursos de la unidad de aprendizaje, a través de ejercicios, } \\
\text { tutoriales, enlaces, textos, etcétera. }\end{array}$ \\
\hline Nivel 4 & $\begin{array}{c}\text { Este nivel suele alcanzarse en las modalidades mixtas, cuyo contenido puede } \\
\text { obtenerse en línea o en forma presencial. Los/as estudiantes generan materiales por sî } \\
\text { mismos/as y proponen recursos o actividades. }\end{array}$ \\
\hline Nivel 5 & $\begin{array}{l}\text { Todo el contenido del curso ocurre en línea, a partir de un enfoque constructivista, en } \\
\text { el cual las interacciones y los contenidos se construyen comunitariamente en línea. }\end{array}$ \\
\hline
\end{tabular}

Nota. Adaptado de J. Silva y M. Romero, 2013, Didasc@lia: Didáctica y Educación, 5(1), $1-22$.

Los entornos virtuales de aprendizaje no siempre alcanzan los niveles más avanzados, de acuerdo a la tipología de Silva y Romero (2015). Sin embargo, desde el año 2000 se ha estado avanzando en un camino más digital, donde el desarrollo de cursos a distancia ha alcanzado un incremento de entre un $80 \%$ y 100\%, a través de una modalidad virtual (Santander, 2012). 
DOCENTE Y APRENDIZ: ENFOQUES EN ESPACIOS VIRTUALES / VALDERRAMA-GACITÚA

La educación virtual ha desentrañado un reto importante: el diseño de currículum, estrategias y objetos de aprendizaje que hagan hincapié en la autogestión del aprendizaje, la interacción, la flexibilidad que demanda el acompañamiento docente asincrónico y la posibilidad de que el estudiante participe en la construcción de su aprendizaje

\section{Docente y aprendiz: enfoques en espacios virtuales}

Los/as docentes avanzan en la enseñanza a distancia, poniendo a prueba todas las posibilidades tecnológicas y didácticas que poseen, en un espacio para el cual muchas veces no están suficientemente capacitados/as. ¿Cómo son esos escenarios? ¿Se logra una conexión en ambientes virtuales entre docente y aprendiz? Algunos investigadores afirman que "la comunicación intergrupal posee un alto componente afectivo y es fundamental para contrarrestar los prejuicios que algunas personas tienen cuando atribuyen a los entornos virtuales una comunicación poco cercana o poco cálida" (Bautista, Borges y Forés, 2006, p.118.)

Estos ambientes virtuales de aprendizaje muchas veces se ven mediados por estrategias arraigadas en la educación presencial, a las que difícilmente se les puede dar continuidad en los entornos digitales. A pesar de ello, dicha continuidad posee elementos afectivos importantes, lo que nos hace preguntarnos: ¿qué estudios han abordado el impacto a nivel afectivo y emocional de avanzar hacia una educación a distancia? Lo anterior, asumiendo que en la presencialidad es muchas veces más fácil monitorear la construcción social. Como indica Marchena,

En el aula de clases se originan relaciones sociales que entablan los protagonistas de una clase, así como por la forma de pensar de cada uno de ellos, por sus valores, esto es, por la cultura existente en el aula (2005, p.198).

Cabe preguntarse, ¿lo anterior se pierde en un medio virtual? Estudios plantean que existen mecanismos para asegurar un aula potencialmente afectiva con ciertas intervenciones a tiempo, como señalan Hernández-Sánchez, Alba y Ortega (2015), quienes plantean la importancia de realizar tutorías afectivas. Ello se condice con lo que afirman Bautista, Borges 
y Forés (2006), respecto a que la comunicación en espacios virtuales tiene componentes de calidez y cercanía, derribando los prejuicios que existen de este modelo de aprendizaje.

Por otra parte, Hernández-Sánchez afirma que "las tutorías afectivas han reforzado los estados emocionales del alumnado y así aumentado el disfrute en el proceso de aprendizaje” (2015, p.21). Esto pone de manifiesto la importancia de una preparación emocional de el/la docente para poder entregar a el/la aprendiz lo que requiere en los momentos indicados, considerando además que "la cantidad de disfrute que los profesores y profesoras obtienen de la docencia se relaciona con la cantidad de atención que los estudiantes muestran" (Rubio y García, 2010, p.98). Esta atención debería estar mediada por el vínculo que se puede desarrollar oportunamente, aun sin tener la relación cara a cara que habitualmente se evidencia en los espacios físicos de educación.

Uno de los elementos esenciales y necesarios en el vínculo docente y aprendiz es la empatía, la cual desempeña un papel central en "la disposición pro-social de las personas" (Mestre, 2004, p.255), dado que "de ésta depende la habilidad para funcionar de manera óptima dentro del contexto social, para lo cual es fundamental comprender lo que sienten los demás” (Moya-Albiol, Herrero y Bernal-García, 2010, p.90). Complementariamente, estudios confirman que "altos niveles de empatía se dirigen a una mayor conducta cooperativa” (Rumble, 2010, p.857) y que las intervenciones que potencian la empatía “incrementan la conducta prosocial altruista" (Rumble, 2010, p.857). En base a esta evidencia ¿cómo generar estos procesos en escenarios virtuales?, ¿el aprendiz logra desarrollar una empatía en medios virtuales?

\section{Empatía en el rol del docente y aprendiz}

Se ha descrito la empatía como "un concepto que incluye aspectos cognitivos, afectivos y emocionales. En lo cognitivo, la empatía involucra la habilidad de entender las experiencias y sentimientos interiores de otra persona, y la capacidad de ver el mundo externo desde la perspectiva del otro" (Hojat et. al., 2002, p. 1563). En el dominio afectivo, la empatía implica la capacidad de unirse a las experiencias y sentimientos de la otra persona. 
La principal ventaja de las estrategias de enseñanza con tecnologías de empatía consiste en fomentar un aprendizaje interactivo, a través de la inmersión en la experiencia, y poner de manifiesto cuáles son las capacidades que posee el/la docente para enfrentar este desafío. Pero, ¿conectarse a un medio virtual de educación afecta la empatía? Si se toman en consideración los elementos de la comunicación no verbal, muchas veces esenciales para leer emociones -como las expresiones faciales, la postura corporal, contacto visual, gestos y tactoy se comparan con textos, mensajes instantáneos y conversaciones en redes sociales, es posible sostener que las experiencias de empatía no serían las mismas.

Sin embargo, de acuerdo a Caplan y Turner (2007), estar en un entorno virtual de aprendizaje puede apoyar la empatía o incluso aumentarla. Estos autores señalan que, a través de la comunicación mediada por un entorno virtual, como las redes sociales, se podría facilitar la empatía, debido al acceso fácil y frecuente a otras personas en situaciones similares. Es decir, cuando todo un medio se entrega bajo las mismas circunstancias, la empatía virtual juega el mismo rol que la empatía real. La importancia de que esta comunicación mediada sea entendida desde el rol del docente, plantea un desafío constante en los ámbitos de la observación del aprendiz y de las diversas habilidades emocionales que este posee. En los últimos años se ha demostrado la importancia de tener en cuenta "las habilidades emocionales y las disposiciones de las personas para comprender lo social y la adaptación emocional“"(Lopes, 2003, p. 653). En este sentido, surge la reflexión sobre cuál es la importancia que tienen las relaciones y competencias sociales dentro del currículum chileno.

Abordar estrategias que desarrollen una formación empática en ambientes virtuales, habla de un gran desafío a nivel país. El proceso de aprendizaje, en cualquier medio que se imparta, es un proceso continuo $\mathrm{y}$, al enfrentarnos a espacios virtuales, estos pueden brindar oportunidades diferentes. Se afirma que "la educación a distancia es un campo emergente, en constante evolución y transformación", donde "la interacción y el diálogo comunicativo" (Martínez, 2008, p.23) son elementos claves para abordar lo que hoy enfrentamos, una realidad educativa que se desarrolla a través de medios digitales. ¿Cómo podemos adaptarlo a nuestras necesidades educativas? Como se mencionó anteriormente, es importante 
REVISTA SABERES EDUCATIVOS, Nº 5, JULIO-DICIEMBRE 2020

considerar los estudios previos y generar adaptaciones de esas intervenciones prácticas para agilizar la gestión educativa desde una mirada de procesos y no de resultados.

Es necesario trabajar en estudios que monitoreen la empatía en medios virtuales y estrategias que la modulen, como la realidad virtual, juegos de roles, entre otros. Desde el papel trascendental que cumplen docentes y aprendices dentro de un espacio formativo y la ganancia de los aprendizajes al existir estas estrategias bien cimentadas, en función de contar con herramientas consistentes a la hora de enfrentar una educación a distancia que esté a la base de las necesidades emocionales que se presentan diariamente.

\section{Referencias}

Bautista, G., Borges, F., y Forés, A. (2006). Didáctica universitaria en entornos virtuales de enseñanza-aprendizaje. Narcea, Madrid, España.

Caplan, S. E., \& Turner, J. S. (2007). Bringing Theory to Research on Computer Mediated Comforting Communication. Computers in Human Behavior, 23(2), 985-998.

Harmon, W. \& Jones, M. G. (1999). The Five Levels of Web Use in Education: Factors to Consider in Planning Online Course. Educational Technology, 39(6), 28- 32.

Hernández-Sánchez, Alba, M., y Ortega, J. A. (2015). Affective e-Learning: An Innovative Model for Developing a Mentoring Virtual Action in an Inclusive Context. Formación Universitaria, 8(2), 19-26.

Hojat, M., Gonnella, J. S., Nasca, T. J., Mangione, S., Vergare, M., \& Magee, M. (2002). Physician Empathy: Definition, Components, Measurement, and Relationship to Gender and Specialty. The American Journal of Psychiatry, 159(9), 1563-1569.

Lopes, P., Salovey, P. \& Straus, R. (2003). Emotional Intelligence, Personality and the Perceived Quality of Social Relationships. Personality and Individual Differences; 35(3): 641- 658 . 
DOCENTE Y APRENDIZ: ENFOQUES EN ESPACIOS VIRTUALES / VALDERRAMA-GACITÚA

Marchena, R. (2005). Mejorar el ambiente en las clases de Secundaria. Un enfoque práctico para responder a la diversidad desde el aula. Aljibe. Málaga.

Martínez, C. (2008). La educación a distancia: sus características y necesidad en la educación actual. Educación, 17(33).

Mestre, V., Frías, M. D., y Samper, P. (2004). La medida de la empatía: análisis del Interpersonal Reactivity Index. Psicothema, 16, 255- 260.

Moya-Albiol, L., Herrero, N., y Bernal-García, M. (2010). Bases neuronales de la empatía. Revista de Neurología, 50(2), 89-100.

Ocampo, A. (2014). Los desafíos de la 'inclusión' en la educación superior latinoamericana en el siglo XXI. Universitaria: Docencia, Investigación e Innovación, 3(2), 65-85.

Santander, W. (18-22 junio 2012). Una visión del estado del arte en la educación superior a distancia en Chile [ponencia]. XIII Encuentro Internacional Virtual Educa, Panamá.

Silva, J. E. y Romero, M. (2013). La virtualidad, una oportunidad para innovar en educación: un modelo para el diseño de entornos virtuales de aprendizaje. Didasc@lia: Didáctica y Educación, 5(1), 1-22.

Rubio, F. y García, C. (2010). La experiencia de fluidez en la educación, en A. Caruana (Coord.), Aplicaciones educativas de la psicología positiva (90-109). Conselleria d'Educació, Generalitat Valenciana, España.

Rumble, A. C. (2010). Empathy Induced Cooperation and Social Dilemmas: An Investigation into the Influence of Attribution Type. Washington State University, Estados Unidos. 